\title{
Identification of a ternary protein-complex as a therapeutic target for K-Ras-dependent colon cancer
}

\author{
Xiaomei $\mathbf{Q i}^{1}{ }^{1,}$, Congying Xie ${ }^{2,}$, , Songwang Hou ${ }^{1}$, Gang $\mathrm{Li}^{2}$, Ning $\mathrm{Yin}^{1}$, Lei Dong ${ }^{2}$, \\ Adrienne Lepp ${ }^{1}$, Marla A. Chesnik ${ }^{3}$, Shama P. Mirza ${ }^{3}$, Aniko Szabo ${ }^{4}$, Susan Tsai ${ }^{5}$, \\ Zainab Basir ${ }^{6}$, Shixiu $\mathbf{W u}^{2,8}$ and Guan Chen ${ }^{1,7}$ \\ ${ }^{1}$ Department of Pharmacology and Toxicology, Medical College of Wisconsin \\ 2 Department of Radiation Oncology, First Affiliated Hospital, Wenzhou Medical College, Wenzhou, China \\ ${ }^{3}$ Department of Biochemistry, Biotechnology \& Bioengineering Center, Medical College of Wisconsin, Milwaukee, WI \\ ${ }^{4}$ Division of Biostatistics, Medical College of Wisconsin, Milwaukee, WI \\ ${ }^{5}$ Department of Surgery, Medical College of Wisconsin, Milwaukee, WI \\ ${ }^{6}$ Department of Pathology, Medical College of Wisconsin, Milwaukee, WI \\ ${ }^{7}$ Research Services, Zablocki Veterans Affairs Medical Center, Medical College of Wisconsin, Milwaukee, WI \\ ${ }^{8}$ The current address at Cancer Center, Hangzhou Cancer Hospital, Zhejiang, China \\ * These authors contributed equally to work \\ Correspondence to: Guan Chen, email: gchen@mcw.edu \\ Shixiu Wu, email: wushixiu@medmail.com.cn \\ Keywords: p38y MAPK, Hsp90, K-Ras, ternary complex, therapeutic target, and colon cancer \\ Received: February 20, $2014 \quad$ Accepted: May 23, $2014 \quad$ Published: May 25, 2014
}

This is an open-access article distributed under the terms of the Creative Commons Attribution License, which permits unrestricted use, distribution, and reproduction in any medium, provided the original author and source are credited.

\section{ABSTRACT}

A cancer phenotype is driven by several proteins and targeting a cluster of functionally interdependent molecules should be more effective for therapeutic intervention. This is specifically important for Ras-dependent cancer, as mutated (MT) Ras is non-druggable and targeting its interaction with effectors may be essential for therapeutic intervention. Here, we report that a protein-complex activated by the Ras effector p38y MAPK is a novel therapeutic target for K-Ras-dependent colon cancer. Unbiased proteomic screening and immune-precipitation analyses identified p38Y interaction with heat shock protein 90 (Hsp90) and K-Ras in K-Ras MT, but not wild-type (WT), colon cancer cells, indicating a role of this complex in Ras-dependent growth. Further experiments showed that this complex requires p38y and Hsp90 activity to maintain MT, but not WT, K-Ras protein expression. Additional studies demonstrated that this complex is activated by $\mathrm{p} 38 \mathrm{y}$-induced Hsp90 phosphorylation at S595, which is important for MT K-Ras stability and for K-Ras dependent growth. Of most important, pharmacologically inhibition of Hsp90 or p38y activity disrupts the complex, decreases K-Ras expression, and selectively inhibits the growth of K-Ras MT colon cancer in vitro and in vivo. These results demonstrated that the p38y-activated ternary complex is a novel therapeutic target for K-Ras-dependent colon cancer.

\section{INTRODUCTION}

Mutated (MT) K-Ras is the most established oncogene in human cancer, which occurs in up to $50 \%$ of colon cancer [1,2]. Despite of extensive studies for more than 40 years, MT K-Ras is still non-druggable and there is a complete lack of targeted therapies against K-Ras MT cancer $[3,4]$. Moreover, cancers with MT K-Ras are more 
aggressive [5] and resistant to targeted therapies such as anti-EGFR (epidermal growth factor receptor) $[6,7]$ and MAPK kinase (MEK) inhibitors [8, 9]. Therefore, there continues to be an urgent need to search for novel targets for therapeutic development against K-Ras MT cancers. Since an activated onco-protein requires interaction with crucial partners for oncogenic activity [10-12], targeting a complex of functionally interdependent molecules may be a strategy for treatment of K-Ras dependent cancer.

Multiple pathway activities contribute to Ras oncogenesis, including MAPK (mitogene-activated protein kinase), PI3K (phosphatidylinosital 3-kinase)/ Akt, and PKC (protein kinase C) cascades [1, 13]. Exact mechanisms by which these kinases regulate Ras oncogenisis, however, have not been demonstrated. p38 MAPKs consist of four family proteins $(\alpha, \beta, \gamma$, and $\delta)$. In contrast to the tumor suppressor role of $\mathrm{p} 38 \alpha$ [1416], p38 $\gamma$ is required for Ras oncogene activity [1720]. In a manner different than the classical MAPK activation by phosphorylation [21], p38 $\gamma$ expression and phosphorylation are both increased in K-Ras MT human colon cancer [22]. Recent studies further showed that p38 $\gamma$ is overexpressed in several types of human cancers such as colon cancer [20, 23], breast cancer [24-26], liver cancer [27], and gliomas [28]. Furthermore, we recently showed that $\mathrm{p} 38 \gamma$ binds and phosphorylates several proteins important for cancer growth and progression including protein tyrosine phosphatase H1 (PTPH1) [22], DNA topoisomerase II $\alpha$ [29], and estrogen receptor [24]. These results indicate that $\mathrm{p} 38 \gamma$ may promote the malignant growth through interacting and phosphorylating its substrates. However, the exact mechanism by which activated p38 $\gamma$ promotes the K-Ras dependent colon cancer growth remains unknown.

Heat shock protein 90 (Hsp90) is a highly conserved molecular chaperone that stabilizes and thereby activates more than 200 client proteins [30]. Hsp90 uses the energy generated by ATP binding/hydrolysis to fold its client protein in a proper structure through cooperation with its co-chaperones [31]. In doing so cancer cells protect an array of mutated and/or overexpressed oncoproteins from misfolding and degradation thereby maintaining the constitutively proliferative signaling $[30,31]$. Indeed, many of Hsp90 clients are proliferative [32-34] and/or transforming $[34,35]$ kinases or activated oncoproteins. Despite the central role of K-Ras in human cancer, a direct connection between K-Ras mutations and Hsp90 signaling has not been reported.

Hsp90 proteins from tumor cells are about 100 -fold more potent in binding to its inhibitor 17-alllylaminogeldanamycin (17-AAG) than those from normal cells [36], indicating a potential of Hsp90 inhibitors in cancer therapy [37, 38]. However, Hsp90 inhibitors are frequently associated with toxicities and poor selectivity, likely as a result of abundant Hsp90 client proteins in cancer cells $[12,38]$. Recent studies showed that Hsp90 phosphorylation can increase its specificity to a certain client without affecting others [39]. Here, we tested the hypothesis that the Ras effector p38 $\gamma$ may drive $\mathrm{K}$-Ras dependent colon cancer growth by activating an oncogenic complex through Hsp90. Our results showed that p38 $\gamma$ specifically forms a complex with Hsp90 and $\mathrm{K}$-Ras in K-Ras MT colon cancer cells through activating Hsp90 by phosphorylation. Disrupting of this ternarycomplex by targeting Hsp90 or p38 $\gamma$ decreases MT K-Ras expression and selectively inhibits K-Ras dependent colon cancer growth in vitro and/or in vivo. These results demonstrated that a ternary protein-complex activated by the Ras effector p38 $\gamma$ is a therapeutic target for K-Ras dependent colon cancer.

\section{RESULTS}

\section{p38 $\gamma$ overexpression in colon cancer correlates with a shortened survival and increased metastasis, and p38 $\gamma$ forms a complex with Hsp90 and K-Ras in K-Ras MT but not WT colon cancer cells}

Our previous studies have shown that $\mathrm{p} 38 \gamma$ is overexpressed in about $80 \%$ of primary colon cancers [20]. Here, we wanted further to examine if overexpressed p38 $\gamma$ has a prognostic activity in clinical colon cancer. In this regard, a group of specimens were first analyzed by immunohistochemistry (IHC) for p38 $\gamma$ expression [20, 23]. To examine the relationship between $\mathrm{p} 38 \gamma$ expression and patients' survival, the overall survival time was plotted according to $\mathrm{p} 38 \gamma$ expression levels $[20,23]$. Results in Figure 1A (left) (Supporting information, Figure 1A) showed that increased p38 $\gamma$ expression correlates significantly with a shortened survival, with the 5-year overall survival rate of $19.8 \%$ in the high $\mathrm{p} 38 \gamma$ group versus $61.8 \%$ in the low p38 $\gamma$ group $(\mathrm{P}<0.05)$. Moreover, levels of p38yexpression are also significantly increased in lymph-node positive colon cancer (Figure 1A, right). These results together indicate that $\mathrm{p} 38 \gamma$ overexpression is a poor prognosis marker for clinical colon cancer. Because elevated $\mathrm{p} 38 \gamma$ is required for K-Ras transformation [17, $20]$, we wanted next to determine if up-regulated p38 $\gamma$ distinctively regulates the growth of colon cancer cells with and without K-Ras mutation through interacting with a different set of proteins.

To explore this possibility, we stably expressed Flag-p38 $\gamma$ in the isogenic cell-line pair HCT116 and Hke3. HCT116 are colon cancer cells with a wild-type (WT) K-Ras and a mutant (MT) K-Ras (K-Ras ${ }^{\mathrm{D} 13}$ ) allele. Somatic deletion of the K-Ras ${ }^{\mathrm{D} 13}$ allele generated Hke3 cells with a reverted oncogenic phenotype in vitro and 


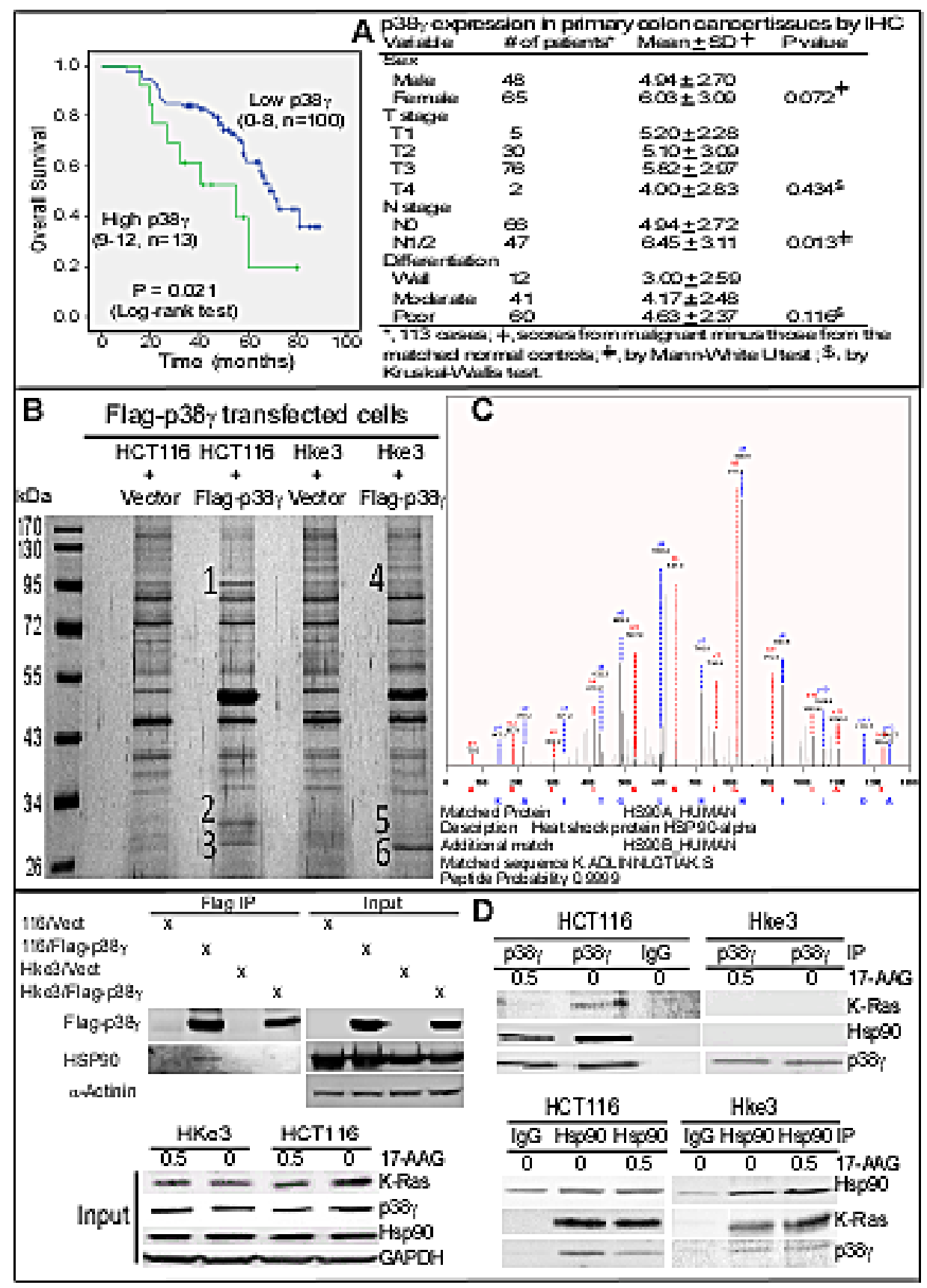

Figure 1: $\mathrm{p38} \gamma$ overexpression in CRC specimens correlates with shortened patient survival and p38 $\gamma$ specifically forms a ternary complex with Hsp90 and K-Ras in K-Ras MT colon cancer cells. (A) Colon cancer specimens were stained for $\mathrm{p} 38 \gamma$ expression by IHC [20] and Kaplan-Meier plot of the overall survival according to $\mathrm{p} 38 \gamma$ levels was shown (left), and associated clinical parameters were given at left. (B, C) Flag immune-precipitates were separated by SDS-PAGE and samples from the numbered region (1-6) were digested, which were then subjected to proteomic analysis as described [22]. MS/MS spectra of heat shock protein $90 \alpha$ peptide HLEINPDHSIIETLR and $90 \beta$ peptide ADLINNLGTIAK with representative peak matches for $\mathrm{b}$ and $\mathrm{y}$ ions \{detected from the sample 1 but not sample 4 of B . Additional proteomic data were given in Supplementary Figure S1B/C). (D) Western blotting (WB)

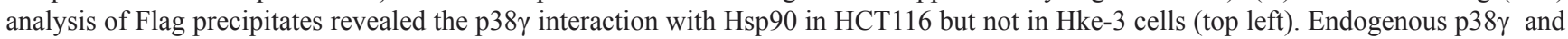
Hsp90 proteins were also isolated after treatment with 17-AAG or DMSO for their interactions with K-Ras (top right and bottom right) with a portion of whole cell lysates analyzed by direct WB as an input (bottom, left). 
in vivo [42]. Flag antibody-isolated precipitates were then subjected to proteomic analysis to screen for MT $\mathrm{K}-$ Ras dependent $\mathrm{p} 38 \gamma$ binding partners [20]. Results in Figure 1B/C (Supporting information, Figure 1B/C) showed that the precipitates from HCT116, but not from Hke3 cells, contained Hsp90, as revealed by detection of three peptides from Hsp90 $\alpha$ protein in which two of them also match to its family member Hsp $90 \beta$ sequence. These results were further confirmed by Western Blot (WB) analysis of Flag precipitates using an antibody reactive both with Hsp90 $\alpha$ and Hsp90 (defined as Hsp90) (Figure 1D, top left), indicating a MT K-Ras dependent Hsp90-binding activity of p38 $\gamma$. Analysis of endogenous p38 $\gamma$ precipitates (Figure 1D, right) further showed that p38y binds Hsp90 and K-Ras in HCT116 but neither in its MT K-Ras disrupted Hke3 line [42, 43]. Although Hsp90 precipitates contain p38 $\gamma$ and K-Ras in both cell lines, an inhibition of Hsp90 activity with 17-AAG only decreases levels of the associated K-Ras and p38y in 116 cells (Figure 1D, bottom right). Significantly, incubation with 17-A significantly down-regulates K-Ras protein expression in whole cell lysates (input) in HCT116, but not Hke3, cells (Figure 1D, bottom left). These results revealed that $\mathrm{p} 38 \gamma$ forms a $17-\mathrm{AAG}$ sensitive ternary complex with Hsp90 and K-Ras only in K-Ras MT cells, indicating its role in transduction of MT K-Ras signaling to Hsp90.

\section{MT K-Ras is an Hsp90 client protein and the Hsp90 inhibitor 17-AAG selectively suppresses K-Ras-dependent colon cancer growth in in vitro and/or in vivo}

Hsp90 plays a central role in protection of mutated and activated onco-proteins from degradation [30]. Its role in protecting MT K-Ras protein, however, has never been demonstrated. The presence of K-Ras protein in the p38 $/$ Hsp 90 precipitates and its down-regulation by $17-$ AAG in HCT116 (but not Hke3) cells suggest that K-Ras may be converted into an Hsp90 client protein upon mutation through $\mathrm{p} 38 \gamma$ actions. To demonstrate if K-Ras mutation alone is sufficient to trigger this conversion, increasing amounts of WT and MT (G12V) K-Ras in pcDNA3 vector were transiently expressed in normal human colon epithelial NCM 460 cells. Cells were then incubated with and without 17-AAG, and analyzed for protein expression by WB. Results in Figure 2A showed that levels of expressed HA-tagged MT K-Ras protein are significantly down-regulated by 17-AAG, whereas those of WT K-Ras are only minimally affected, indicating that $\mathrm{K}-\mathrm{Ras}$ is converted into an Hsp90 client protein upon mutation. To further demonstrate if endogenous MT $\mathrm{K}-\mathrm{Ras}$ is an Hsp90 client, colon cancer cells harboring WT or MT K-Ras [20] were treated with 17-AAG and effects on the Ras expression were analyzed by WB. Results in
Figure 2B showed that incubation with 17-AAG only down-regulates K-Ras in K-Ras MT cells but decreases Raf-1 \{an established Hsp90 client [32]\} in all cell lines. These results, for the first time, demonstrated that both transfected and endogenous MT K-Ras is an Hsp90 client protein.

To investigate if Hsp90 may act as a therapeutic target for K-Ras MT colon cancer, HCT116 and its two MT K-Ras disrupted sub-lines [42, 44] were incubated with 17-AAG and effects on cell-death were determined. Results in Figure 3A showed a 17-AAG dose-dependent decrease in cell viability in HCT116, but not in Hke3 and HK2-8, cells. Moreover, a re-expression of the MT $\mathrm{K}-\mathrm{Ras}(\mathrm{G} 12 \mathrm{~V})$ in Hke3 cells increases p38 $\gamma$ expression and enhances the sensitivity to 17-AAG (Supporting information, Figure S2A). These results indicate a sufficient role of MT K-Ras in inducing p38 $\gamma$ expression and in increasing the 17-AAG induced growth-inhibition in human colon cancer cells. An increased toxicity to 17AAG was further demonstrated by a long-term colony formation assay in two MT K-Ras colon cancer cell lines as compared with WT K-Ras Caco2 cells (Figure 3B). To further demonstrate the therapeutic activity of 17-AAG in K-Ras MT colon cancer in vivo, HCT116 and Ls-174T cells were injected into nude mice and the inhibitor was systemically administrated after tumors became palpable. Results in Figure 3C/D (and Supporting information, Figure S2B) showed that 17-AAG significantly inhibits the growth of both xenografts without significant effects on mouse body weight (data not shown). Furthermore, analyses of immune-precipitates from tumor lysates by an antibody against p38 $\gamma$, Hsp90 or K-Ras revealed that there is a ternary-complex formation, which is disrupted by the 17-AAG treatment in both tumors, leading to a downregulation of K-Ras expression (Figure 3E/F). These results together demonstrated that MT K-Ras is an Hsp90 client protein and Hsp90 may be a novel therapeutic target for K-Ras-dependent colon cancer through a complex formation with p38 $\gamma$ and K-Ras.

\section{p38 $\gamma$ activates Hsp90 by inducing its phosphorylation at Ser595, which is important both for K-Ras protein expression in K-Ras MT cells and for $K-R a s$ dependent colon cancer growth}

Hsp90 activity is regulated by phosphorylation [45]. Because levels of p-p38 $\gamma$ protein expression are associated with those of its substrate protein tyrosine phosphatase H1 phosphorylation (p-PTPH1) in K-Ras MT, but not WT, colon cancer cells [22], we next examined if p38 $\gamma$ may phosphorylate Hsp90 thereby increasing its chaperone activity toward MT K-Ras. In this case, Flagtagged human Hsp90 $\alpha$ [39] was transiently expressed in 293T cells and Flag precipitates were incubated with His- 
tagged p38 $\gamma$ in vitro by including His-p38 $\alpha$ for comparison [22]. Phosphorylated Hsp90 proteins were separated in SDS-PAGE and detected with a specific phospho-MAPK substrate antibody ( $p-\mathrm{S} / \mathrm{TP}$ ) [46]. Results in Figure 4A showed that there is an increased phosphorylated band at about $90 \mathrm{kDa}$ after incubation His-p38 $\gamma$, whereas Hisp38 $\alpha$ addition has little effect, indicating that Hsp90 is specifically phosphorylated by $\mathrm{p} 38 \gamma$ in vitro. To demonstrate if $\mathrm{p} 38 \gamma$ phosphorylates Hsp90 in vivo, FlagHsp90 $\alpha$ was co-transfected with a constitutively active

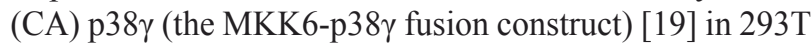
cells. Cells were then incubated with or without a specific p38 $\gamma$ pharmacological inhibitor pirfenidone (PFD) [47] and analyzed for Hsp90 phosphorylation. Results in Figure 4B showed that levels of phosphorylated Hsp90 (p-Hsp90) are significantly increased by the CA p38 $\gamma$ transfection, which, however, is completely blocked by incubation with PFD. The increase and the decrease in p-Hsp90 expression by the $38 \gamma$ activation and inhibition are also associated with up- and down-regulation of the total Flag-Hsp90 protein expression (Figure 4B), indicating that $\mathrm{p} 38 \gamma$ may increase Hsp90 protein expression by phosphorylation. The p-Hsp90 band in the absence of the transfected FlagHsp90 (Figure 4B, lane 1 from right) may result from the endogenous Hsp90. These results together demonstrate that $\mathrm{p} 38 \gamma$ phosphorylates Hsp90 in vitro and in vivo.

The sequence analysis showed that human Hsp90 $\alpha$ contains a single S/TP site (Ser595), a typical
MAPK phosphorylation motif [21]. To explore if p387phosphorylates Hsp90 at this site, a mutated Hsp90/ S595A was generated by site-directed mutagenesis using the PCR-based technique [24]. The WT and MT Hsp90 constructs were then co-transfected with Flag-p38 $\gamma$ in $293 \mathrm{~T}$ cells, which were examined for $\mathrm{p}-\mathrm{Hsp} 90$ expression [46]. Results (Supporting information, Figure 3A, left) showed that p38 $\gamma$ only phosphorylates WT Hsp90 but not Hsp90/S595A mutant even though two proteins are expressed at a similar level, indicating that $\mathrm{p} 38 \gamma$ phosphorylates Hsp90 at S595. The lack of an elevation of the exogenously expressed Hsp90 after its phosphorylation may be due to low levels of Hsp90/S595 phosphorylation as a result of Flag-p38 $\gamma$ (instead of its CA form) used in the co-transfection (Figure 4B vs. Supporting information, Figure S3A). Importantly, stable expression of Hsp90/ S595A in two K-Ras MT colon cancer cell lines decreases endogenous K-Ras expression as compared to the WT Hsp90 transfection and confers a resistance to 17-AAGinduced inhibition of colony formation and cell growth (Supporting information, Figure S3A-C). These results together demonstrated a critical role of the p38 $\gamma$-induced Hsp90/S595 phosphorylation in MT K-Ras protein expression and in K-Ras-dependent colon cancer growth.

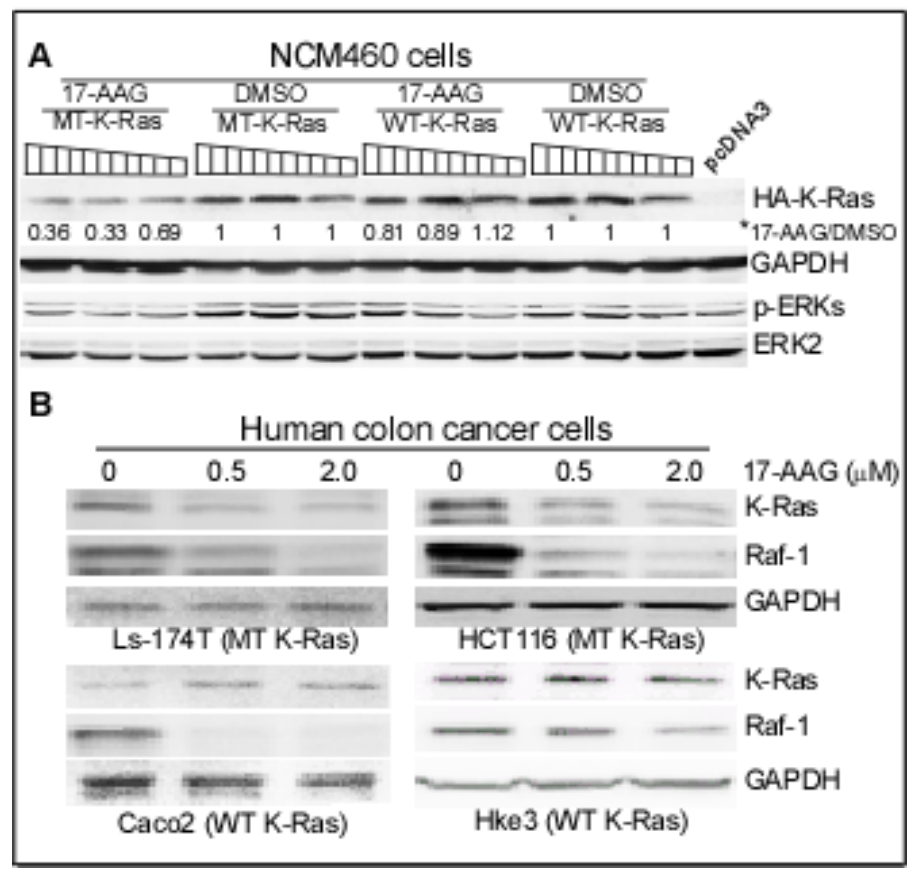

Figure 2: K-Ras is converted to an Hsp90 client protein upon mutation. (A) NCM460 cells were transiently expressed with increasing concentrations $(0.5,1.0$, and $1.5 \mu \mathrm{g}$ DNA) of WT and MT (G12V) K-Ras constructs for 24h, followed by incubation with 0.1 $\mu \mathrm{M}$ of $17-\mathrm{AAG}$ or DMSO for additional $24 \mathrm{~h}$, which were then subjected to WB analysis. *, the band intensity was quantitated by the NIH software, which was normalized with GAPDH, and K-Ras protein level in 17-AAG-treated group was expressed as relative to that treated with DMSO. A separate experiment showed similar results. (B) Colon cancer cells with indicated K-Ras phenotype were incubated with indicated concentration of 17-AAG for $24 \mathrm{~h}$, which were then analyzed for protein expression by WB. Similar results were obtained in a separate experiment. 
The p38 $\gamma$ inhibitor pirfenidone (PFD) preferentially inhibits the colony-formation in K-Ras MT colon cancer cells and synergizes with 17-AAG to specifically inhibit the K-Ras dependent growth in vitro

Previous studies showed that PFD, an antiinflammatory agent [48], specifically inhibits p38 $\gamma$ (but not $\mathrm{p} 38 \alpha$ or $\mathrm{p} 38 \beta$ ) kinase activity in vitro [47]. Consistent with this report, we recently showed that PFD efficiently blocks the p38 $\gamma$ phosphorylation of PTPH1 at S459 in colon cancer cells [22]. Because levels of phosphorylated p38 $\gamma$ protein expression are up-regulated in K-Ras MT cells [20] and PFD efficiently inhibits the p38 $\gamma$-induced
Hsp90 phosphorylation (Figure 4B), we next examined if PFD more effectively inhibits the K-Ras dependent growth. Of great interest, results in Figure 4C showed a greater inhibition of colony formation by PFD in two MT K-Ras cell lines than in their WT K-Ras counterparts. The growth-inhibitory effects of PFD in K-Ras MT cells are similar to those observed by p38 $\gamma$ knockdown using shRNA [20]. Because p38 $\gamma$ phosphorylates and activates Hsp90, and Hsp90 stabilizes MT (but not WT) K-Ras protein (Figure 2/4A/4B), we next explored if PFD may have a synergistic growth-inhibitory activity with 17-AAG depending on K-Ras mutation through their respective inhibition of $\mathrm{p} 38 \gamma$ and Hsp90 activity in the ternary complex. Of great interest, results in Figure 4D (and Supporting information, Figure S4) indeed showed that a

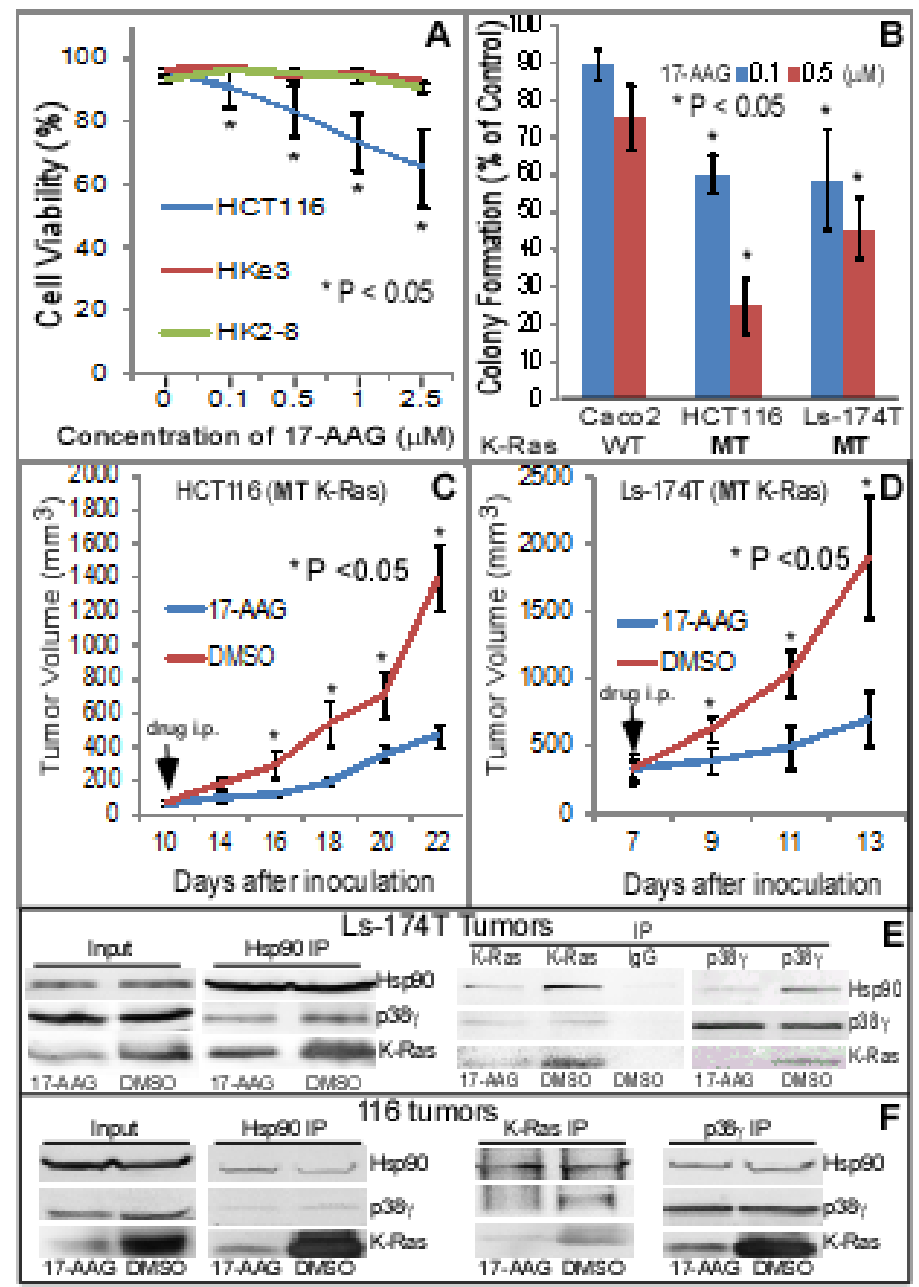

Figure 3: Hsp90 is a therapeutic target for K-Ras MT colon cancer. (A) HCT116 and its MT K-Ras disrupted sub-lines were incubated with 17-AAG for $24 \mathrm{~h}$ and assessed for cell viability using trypan blue staining [22]. Results are means of three experiments (+ $\mathrm{SD}, *$ vs. either Hke3 or HK2-8 cell line). (B) Indicated cells were cultured in the presence and absence of 17-AAG for about 2 weeks and colony formed was manually counted. Results are expressed as $\%$ of solvent treated control (mean $\pm \mathrm{SD}, \mathrm{n}=3, *$ vs. Caco2 cells). (C, D) Indicated human colon cancer cells $\left(2 \times 10^{6} \mathrm{in} 0.1 \mathrm{ml}\right.$ of PBS) were s.c. injected into nude mice on both sides of front flank and 17-AAG $(80 \mathrm{mg} / \mathrm{kg}$ ) or solvent (DMSO) solution (in $50 \mu$ l) was i.p. administrated daily for 14 days for HCT116 tumors and for 6 days for Ls-174T xenograft. Changes in tumor volume and body weight were monitored every other day [20]. Results in all groups are means of 4-5 tumors ( \pm SEM, $\mathrm{n}=4-5$ mice, * vs. DMSO). (E, F) The same amounts of lysate proteins from DMSO or 17-AAG treated tumors were immuneprecipitated with indicated antibodies and precipitates were then analyzed by WB for their complex formation, with a portion of whole lysates analyzed by direct WB as an input. 
combination of PFD with 17-AAG has a greater growthinhibitory effect than either alone in K-Ras MT, but not WT, colon cancer cells. These results together indicate that a combined targeting Hsp90 and its activator p38 $\gamma$ may be a more effective therapeutic strategy against K-Ras dependent colon cancer.

\section{p38 $\gamma$ activity is required for MT, but not WT, K-Ras protein expression through a complex formation with Hsp90}

We have shown that p38 $\gamma$ phosphorylates Hsp90 (Figure 4A/B) and expression of the p38 $\gamma$ nonphosphorable Hsp90/S595A construct or application of the Hsp90 inhibitor 17-AAG decreases MT-K-Ras protein expression (Figure 2B and Supporting information, Figure S3A, right). These results indicate that $\mathrm{p} 38 \gamma$ may increase MT, but not WT, K-Ras protein expression by a phosphorylation-dependent mechanism. To demonstrate this possibility, 293T cells were transiently transfected with Flag-p38 $\gamma$ together with and without HA-tagged WT and MT K-Ras, and resultant effects on the ectopic
Ras protein expression were examined by WB. Results (Supporting information, Figure 5A) showed that p38 $\gamma$ significantly increases the MT K-Ras expression, whereas it only minimally impacts the WT K-Ras level. Moreover, analysis of HA immune-precipitates showed that only MT, but not WT, K-Ras binds transfected Flag-p38\%, but not its AGF mutant (Supporting information, Figure 5B). These results together indicate that $\mathrm{p} 38 \gamma$ increases MT K-Ras expression through a complex formation by a phosphorylation-dependent mechanism.

To investigate if endogenous $\mathrm{p} 38 \gamma$ is also required for MT K-Ras protein expression, colon cancer cells were depleted of $\mathrm{p} 38 \gamma$ by retroviral mediated shRNA expression [20], which were then examined for K-Ras protein expression by WB. Results in Figure $5 \mathrm{~A}$ showed that p38 $\gamma$ depletion decreases K-Ras expression only in cells expressing a MT K-Ras. Moreover, the down-regulated K-Ras by 17-AAG and p38 $\gamma$ shRNA was both reversed by treatment with MG132, a proteasome inhibitor (Figure 5B), indicating that $\mathrm{p} 38 \gamma$ and Hsp90 act by the same proteasome-dependent mechanism to protect MT K-Ras from degradation. In addition, incubation with PFD or

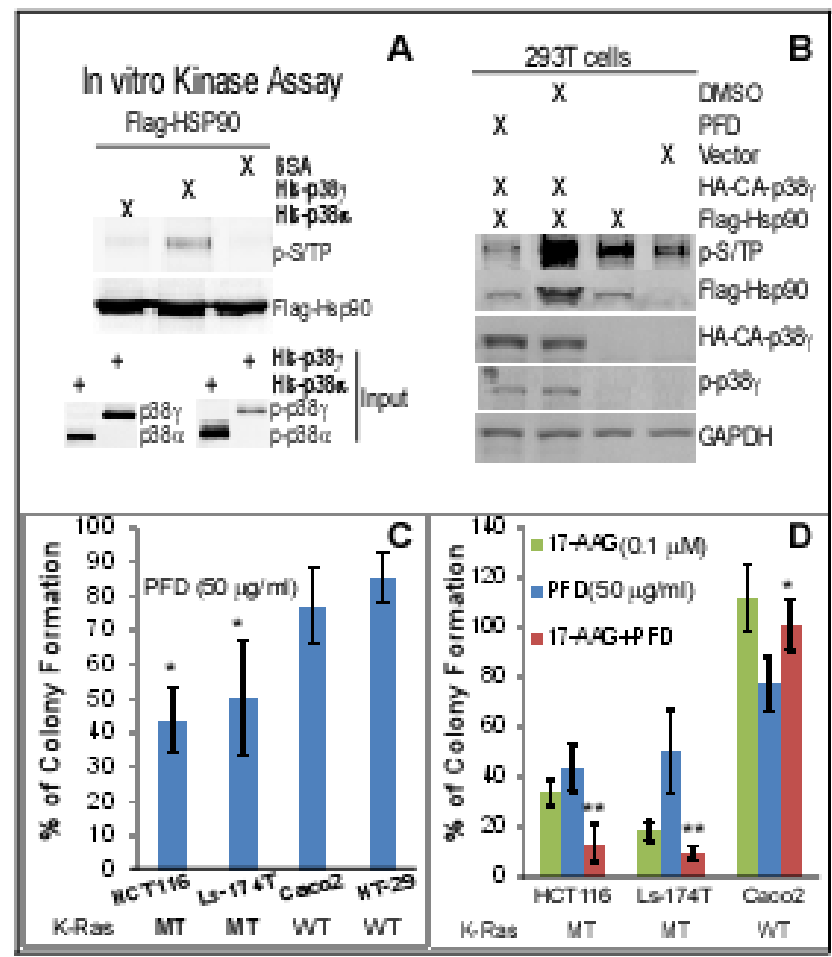

Figure 4: p38 $\gamma$ phosphorylates Hsp90, and the p38 $\gamma$ pharmacological inhibitor pirfenidone (PFD) blocks Hsp90 phosphorylation, more effectively inhibits the K-Ras dependent growth in vitro, and selectively synergizes with 17AAG to inhibit the colony formation of K-Ras MT colon cancer cells. (A) Flag-Hsp90 proteins isolated from 293T cells were incubated with bacterially expressed His-p38 $\gamma$ or His-p38 $\alpha$, and kinase activity assays were performed [24]. Phosphorylated Hsp90 was detected by WB using a specific phospho-MAPK substrate (p-S/TP) antibody (left, top) and the same amounts of His-p38 $\alpha$ and His-p38 $\gamma$ proteins were analyzed by WB as input controls (left, bottom). (B) Indicated constructs were transfected into $293 \mathrm{~T}$ cells for $48 \mathrm{~h}$, with $20 \mu \mathrm{g} / \mathrm{ml}$ of PFD or solvent added for the last $24 \mathrm{~h}$, and cells were then analyzed for protein expression and phosphorylation (right). (C, D) Colon cancer cells were incubated with indicated concentrations of PFD and/or 17-AAG for about 2 weeks. Colonies formed were manually counted and presented as $\%$ of solvent treatment. Results are mean of 3-4 experiments $( \pm \mathrm{SD}, * \mathrm{P}<0.05$ vs. either WT K-Ras line for $\mathrm{C}$; ** $\mathrm{P}<0.05$ vs. either inhibitor, * $\mathrm{P}>0.05$ vs. either inhibitor for $\mathrm{D})$. 
17-AAG decreases the ectopically expressed MT K-Ras protein expression (Supporting information, Figure S5C). These results together indicate that $\mathrm{p} 38 \gamma$ and Hsp90 both protect MT K-Ras protein from proteasome-dependent degradation.

\section{PFD inhibits the xenograft-growth of K-Ras dependent colon cancers in nude mice, disrupts the $\mathbf{p 3 8}$ /Hsp90/K-Ras complex, and decreases MT K-Ras protein expression in tumor tissues}

PFD is a non-toxic and orally active anti-fibrotic agent, and is currently in phase III clinical trials for treatment of lung fibrosis [49-52]. Of great interest, we showed that PFD inhibits p38 $\gamma$ phosphorylation of Hsp90 (Figure 4B), decreases MT K-Ras protein expression (Supporting information, Figure S5C), and selectively suppresses the K-Ras dependent growth in cell culture (Figure 4C/D). We wanted next to determine if PFD has a therapeutic activity against K-Ras MT colon cancer in mice and whether it can target the p38 $/$ Hsp90/K-Ras complex in tumor tissues. In this regard, HCT116 and Ls-174T cells were injected into nude mice, which then received the daily i.p. therapy with PFD or solvent DMSO. Results in Figure 6B showed that the treatment with PFD almost completely blocked the HCT116 xenograft growth. Despite the rapid growth of the Ls-174 tumor likely as a result of its inoculation in Matrigel, the PFD treatment also led to a significant growth inhibition at every time point after the drug administration (Figure 6A). In both cases, there were no drug-associated toxicities. These results demonstrated a strong growth inhibitory activity of the non-toxic p38 $\gamma$ inhibitor PFD in two K-Ras MT colon cancer xenografts.

To demonstrate if PFD also regulates the complex formation, lysates were prepared from tumors and analyzed by Hsp90/p38 $\gamma$ IP/WB. Results in Figure 6C/D showed that the PFD treatment decreases the Hsp90 binding activity both with $\mathrm{p} 38 \gamma$ and K-Ras, and similar results were also obtained when $\mathrm{p} 38 \gamma$ immune-precipitates were analyzed. Levels of total and phosphorylated PTPH1 protein expression were decreased in total tumor lysates by the PFD treatment in both tumors (Figure 6C/D), indicating that the $\mathrm{p} 38 \gamma$ activity is inhibited leading to a decreased PTPH1 phosphorylation and expression [22]. Different than the 17-AAG therapy (Figure 3), the PFD treatment also decreases Hsp90 expression in total

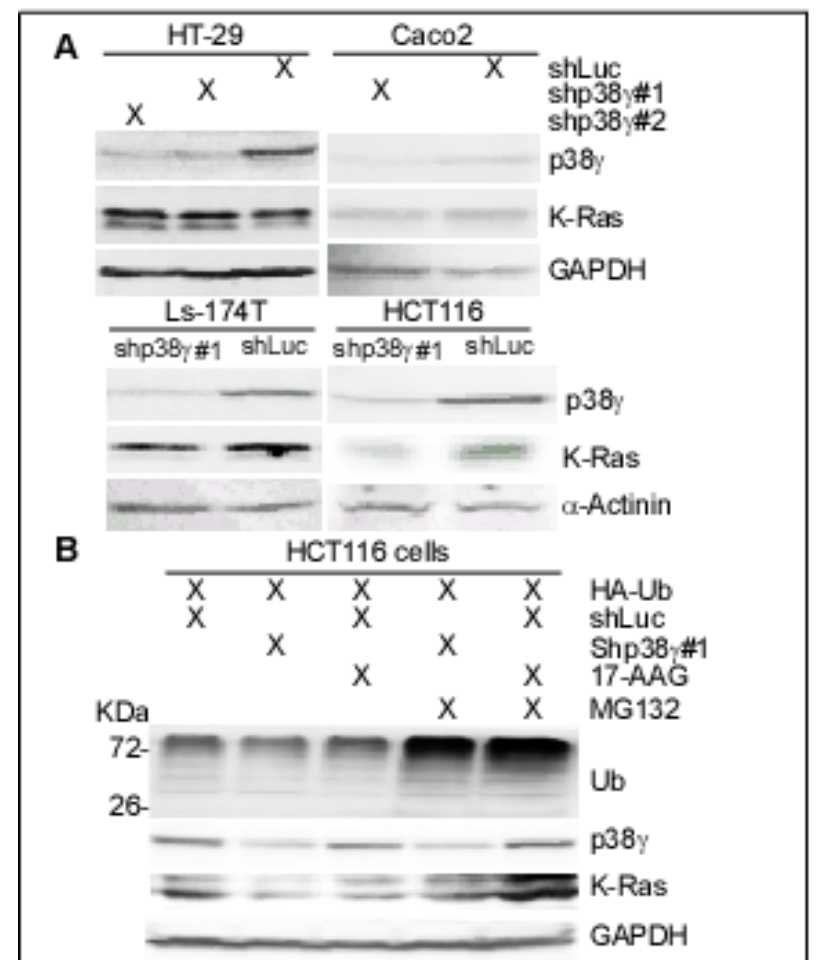

Figure 5: p38 $\gamma$ depletion selectively depletes K-Ras protein in K-Ras MT colon cancer cells. (A) Colon cancer cells were stably depleted of endogenous p38 $\gamma$ protein by lentiviral mediated shRNA delivery [20] and analyzed for protein expression by WB. Similar results were obtained in a separate experiment. (B) HCT116 cells with and without p38 $\gamma$ depletion were transiently transfected with HA-UB for $48 \mathrm{~h}$ with and without incubation with 17-AAG $(0.1 \mu \mathrm{M})$ for the last $24 \mathrm{~h}$, followed by the last 6-h incubation with and without MG132 $(100 \mu \mathrm{M})$. Cell lysates were collected for WB analyses. (C) Colon cancer cells were incubated with $100 \mu \mathrm{g} / \mathrm{ml}$ of PFD or solvent DMSO for $24 \mathrm{~h}$, and then analyzed for protein expression by WB. Similar results were obtained in a separate experiment. 
tumor lysates as observed in $293 \mathrm{~T}$ cells (Figure 4B), indicating that it may inhibit the Hsp90 phosphorylation in tumors, which was confirmed by WB analysis of Hsp90 precipitates (Figure 6C/D). Most importantly, levels of K-Ras protein expression in the Hsp90 and p38 $\gamma$ complexes as well as in whole tumor lysates were all significantly down-regulated by the PFD treatment in both tumors. These results are similar to those obtained after the treatment with the Hsp90 inhibitor 17-AAG (Figure 3E/F). Therefore, targeting the p38 $/ \mathrm{Hsp} 90 / \mathrm{K}$ Ras ternary complex by a p38 $\gamma$ or an Hsp90 inhibitor led to a similar growth-inhibition in K-Ras MT colon cancer by mechanisms converged at down-regulating MT K-Ras protein expression.

\section{DISCUSSION}

$\mathrm{K}$-Ras mutation is the most frequently oncogenic event in human cancer. However, thus far there have been no therapeutic strategies effective against K-Ras dependent malignancies [2]. Here, our studies provide several pieces of evidence that together indicate that a p38 $\gamma$-activated ternary-complex with Hsp90 and K-Ras may be a novel therapeutic target for K-Ras dependent colon cancer. First, unbiased proteomic screening identified Hsp90 as a MT K-Ras dependent binding partner of $\mathrm{p} 38 \gamma$ (Figure 1B/C), an established Ras effector that is overexpressed in primary colon cancer [20] and has a poor prognostic activity (Figure 1A). The complex was further shown to contain K-Ras protein and a pharmacological inhibitor of p38 $\gamma$ or Hsp90 selectively down-regulates K-Ras expression in K-Ras MT cells (Figure 4C/2/5). Additional experiments showed that p38 $\gamma$ activates Hsp90 by direct phosphorylation and both p38 $\gamma$ and Hsp90 maintain K-Ras expression in K-Ras MT cells via inhibition of proteasome-dependent degradation
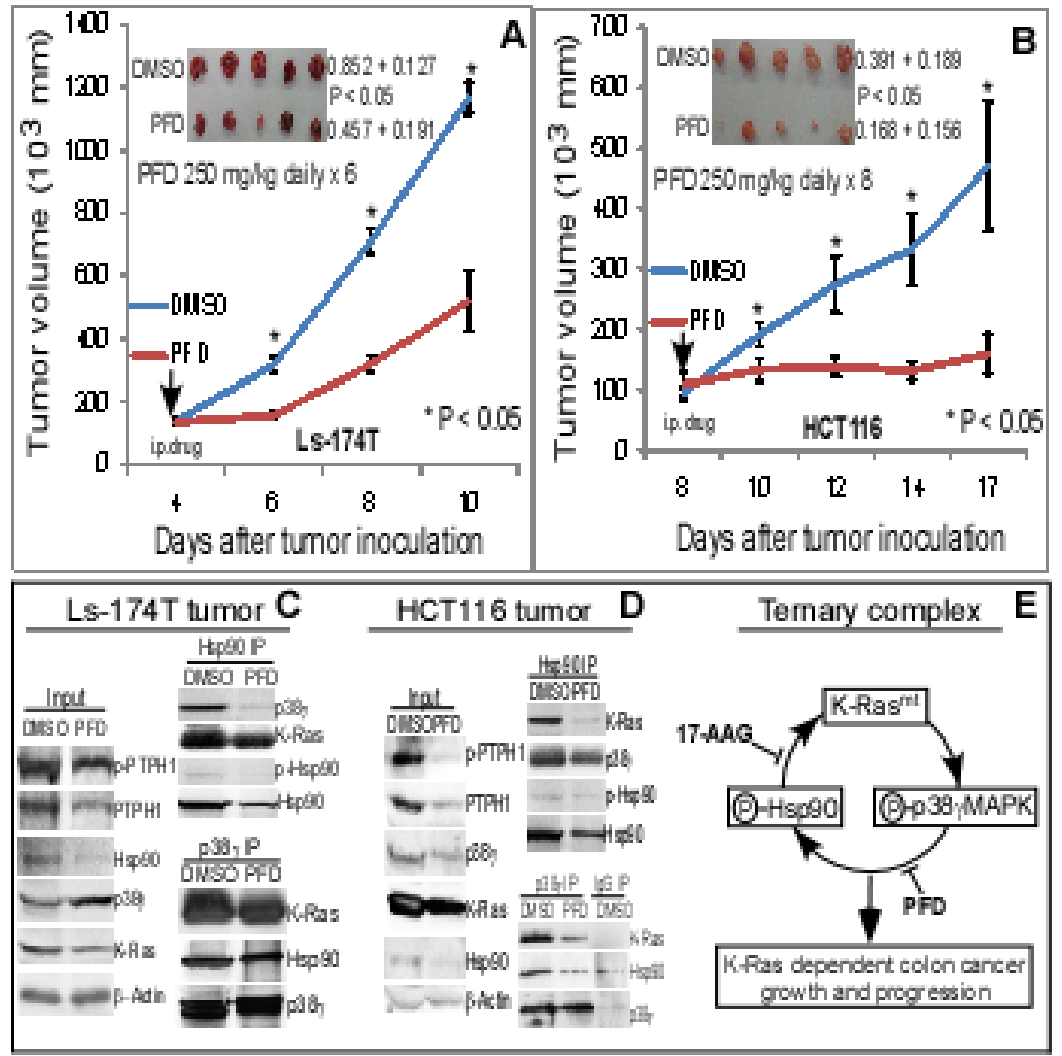

Figure 6: PFD inhibits K-Ras-dependent colon cancer growth in mice, disrupts the p38 $\gamma / \mathrm{Hsp90/K-Ras} \mathrm{complex,} \mathrm{and}$ depletes K-Ras protein in tumor tissues. (A, B) Tumor cells were s.c. injected as described above and daily therapy with PFD or DMSO control was i.p. administrated into mice when tumor becomes palpable. Tumor volume was measured every other day and results shown are mean of 4-5 tumors ( \pm SEM). Tumors were also weighted at the end of experiments (inserts, mean \pm SD, * vs. PFD). (C, D) PFD disrupts the ternary protein-complex and decreases K-Ras protein expression in tumor tissues. The same amount of lysate proteins from PFD or DMSO treated tumor were subjected to IP with indicated antibodies and precipitates were analyzed for complex formation by WB with a portion of total lysates as an input control. (E) An experimental model elucidates that K-Ras mutation stimulates p38 $\gamma$ expression/ phosphorylation leading to Hsp90 phosphorylation resulting in the K-Ras stabilization by a ternary complex. An inhibition of $\mathrm{p} 38 \gamma$ activity by PFD and/or Hsp90 by 17-AAG will attenuate the complex formation, decrease the K-Ras expression, and suppress the K-Ras dependent growth and progression. 
(Figure 4/5). Moreover, the Hsp90 inhibitor 17-AAG and the p38 $\gamma$ inhibitor PFD are both more effective in inhibition of K-Ras MT colon cancer growth in vitro and their combination only has a synergistic growthinhibitory activity in colon cancer cells harboring a MT K-Ras (Figure 3/4). Most significantly, both 17-AAG and PFD significantly inhibit K-Ras dependent growth in mice and attenuate the $\mathrm{p} 38 \gamma / \mathrm{Hsp} 90$ binding with K-Ras leading to K-Ras protein depletion in K-Ras MT tumor tissues (Figure 3/6). These results together demonstrate that a ternary complex activated by the Ras effector $\mathrm{p} 38 \gamma$ is a therapeutic target for K-Ras MT colon cancer (Figure 6E).

Targeting several proteins at the same time by different means for therapeutic intervention has been previously explored $[37,53]$. Our results, however, for the first time, demonstrated that an interdependent proteincomplex initiated by MT K-Ras, activated by its effector p38 $\gamma$, and coordinated and enhanced by Hsp90 acts as a functional oncogene and thus a valid therapeutic target for $\mathrm{K}-\mathrm{Ras}$ mutated colon cancer (Figure 6E). This conclusion is based on the fact that immune-precipitates of either protein from the ternary complex contained the other two (Figure 1D/3E/3F/6C/6D). In this complex, K-Ras activating mutation may act as a driver, which leads to p38 $\gamma$ overexpression [17, 20] (Supporting information, Figure 2A) [22]. Activated p38 yin the complex may act as an activator to facilitate MT K-Ras to become an Hsp90 client protein by activating Hsp90 through phosphorylation thereby sustaining MT K-Ras expression and maintaining K-Ras constitutively proliferative signaling through a complex formation (Figure 3/4). This is because 1) p38 $\gamma$ overexpression increases and its depletion decreases MT, but not WT, K-Ras expression (Figure 5A and Supporting information, Figure S5B); 2) the stimulating effect of p38 $\gamma$ on MT K-Ras protein expression couples with their binding activity with Hsp90 by a mechanism depending on p38 $\gamma$ phosphorylation and K-Ras mutation (Figure 1D and Supporting information, Figure S5A/B); 3) p38 $\gamma$ phosphorylates Hsp90 in vitro and in vivo and an Hsp90 mutant that cannot be phosphorylated by p38 $\gamma$ also inhibits MT K-Ras expression and attenuates the K-Ras dependent growth (Figure 4A/B and Supporting information, Figure S3); 4) the p38 $\gamma$ inhibitor PFD blocks the Hsp90 phosphorylation (Figure 4B), disrupts the K-Ras/p38 $\gamma /$ Hsp90 complex, depletes MT K-Ras protein expression (Figure 6C/D), and preferentially inhibits K-Ras MT colon cancer cell growth (Figure 4C); and 5) PFD only synergizes with 17-AAG to inhibit the K-Ras dependent growth in vitro (Figure 4D and Supporting information, Figure S4). Phosphorylated Hsp90 in the complex, on the other hand, may be an executor to maintain MT K-Ras protein in a proper conformation thereby preventing it from proteasome-dependent degradation (Figure 5B). Although Hsp90 can protect many onco-proteins from degradation [30] such as Bcr-Able [54], Raf-1 [55] and mutated B-Raf [35], its specific MT K-Ras protective

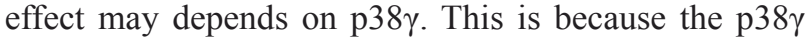
inhibitor PFD both blocks Hsp90 phosphorylation and down-regulates MT K-Ras expression (Figure 4B/6C/D). Therefore, three proteins in this ternary-complex are interdependent with a common signaling output to specifically sustain MT K-Ras protein expression and to selectively promote the K-Ras dependent growth and progression (Figure 6E).

K-Ras can be targeted by specific antisense oligos [56] or miRNA [57] but whether these approaches can specifically target MT K-Ras remains unknown. Our results reported here may indicate a novel therapeutic strategy against K-Ras-dependent malignancies, as targeting either p38 $\gamma$ or Hsp90 decreases MT, but not WT, K-Ras expression and effectively inhibit K-Ras MT colon cancer growth (Figure 1-6). However, we should be cautious about the specificity and efficacy of targeting Hsp90 alone, as Hsp90 can also act on the MT tumor suppressor p53 [58] and Hsp90 inhibition can induce Hsp70 thus decreasing the therapeutic efficacy [59]. But, our results of the combination strategy of the p38 $\gamma$ inhibitor PFD with the Hsp90 inhibitor 17-AAG to synergistically and selectively inhibit the K-Ras dependent growth (Fig. 4d) are very promising and may have a great application potential. While these inhibitors may have off-target effects, our results strongly indicate a cooperative role of p38 $\gamma$ and Hsp90 in maintaining MT $\mathrm{K}$-Ras expression and consequently in driving the K-Ras dependent growth. Although the therapeutic advantages of PFD in combination with 17-AAG over either alone require further experimentations in mice to rule out potential synergistic toxicities in vivo, these results provide a proof of concept that a combined targeting the two steps in the complex can achieve more effective therapeutic effects against K-Ras dependent colon cancer. Previous studies showed that Hsp90 stabilizes the serine/ threonine kinase STK33 leading to an increased sensitivity of K-Ras MT cancer cells to Hsp90 inhibitors but whether this kinase has any effect on the Ras oncoprotein or Hsp90 has not been demonstrated [60]. Moreover, while several Hsp90 inhibitors are currently in clinical trials against several types of human malignancies, their poor selectivity and severe toxicities have become a main concern [12]. Addition of the p38 $\gamma$ inhibitor PFD to the therapeutic regime with an Hsp90 inhibitor may increase its selectivity and/or reduce its toxicities. Most importantly, PFD is non-toxic [51] and alone is more effective in inhibiting $\mathrm{K}$-Ras MT than WT colon cancer growth in vitro and/or in vivo (Figure 4/6). Therefore, this FDA approved antifibroblastic drug may have immediate benefits in its new application against K-Ras MT colon cancer. 


\section{MATERIALS AND METHODS}

\section{Constructs, cell lines, antibodies, inhibitors, and other reagents}

His-tagged p38 $\gamma$ and p38 $\alpha$ as well as Flag-tagged p38 $\gamma$ expressing constructs were provided by Dr. Jiahuai Han [40]. Flag-tagged human Hsp90 $\alpha$ was a gift from Dr. Len Neckers [41], whereas retrovirus LZRS-K-Ras and the MKK6-p38 $\gamma$ constructs have been described previously $[17,19,20]$. WT and MT (G12V) K-Ras in HA-tagged pcDNA3 vector were kindly provided by Dr. Carol Williams or purchased from Guthrie cDNA resource center. The pLenti6/Block-iT system (Block-it ${ }^{\mathrm{TM}} \mathrm{U} 6 \mathrm{RNAi}$ entry vector Kit, Cat: K4944-00 and Block-it ${ }^{\mathrm{TM}}$ Lentiviral RNAi expression system, Cat: K4943-00, Invitrogen) was used to clone sequences for shRNAs. The target sequences for individual genes are as following; luciferase (shLuc): GTGCGTTGCTAGTACCAAC (shLuc); human p38\%:

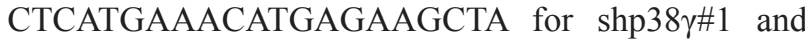
GAAGGAGATCATGAAGGTGAC for shp38y\#2 [20]. Human colon cancer cell lines CaCo2, HT29, Ls-174T were purchased from ATCC and normal human colon epithelial NCM460 cells were bought from INCELL Corporation. HCT116 and its MT K-Ras disrupted sublines (Hke3 and HK2-8) were provided by Dr. Shirasawa [42]. Hsp90 protein inhibitor 17-AAG (geldanamycin) was from Selleck Chemicals (Cat. Number: S1141), whereas p38 $\gamma$ inhibitor pirfenidone (PFD) was purchased from Sigma (Product Number: P2116). Both drugs were dissolved in DMSO, which were stored at $-20^{\circ} \mathrm{C}$ until use. The mouse phospho-antibodies (p-p38, p-ERK, p-JNK) and phosphorylated MAPK substrate (p-S/TP, Cat \#:2325) were purchased from Cell Signaling. Other antibodies were provided by Santa Cruz, including mouse anti-KRas (sc-30), rabbit an-Hsp90 (sc-7947), mouse anti-Flag (sc-807), mouse anti-GAPDH (sc-47724), mouse anti-HA (sc-7392), mouse anti- $\alpha$-Actinin (sc-17829) and rabbit anti-p38 $\alpha$ (sc-535). Anti-p38 $\gamma$ goat and rabbit antibodies were bought from R\&D Systems. Serum and other cell culture materials were from Gibco and all other chemicals were from Sigma.

\section{Proteomic analysis}

The SDS-PAGE gel containing the proteins of interest as indicated (Fig. 1B) was cut, which was used for in gel digestion, $\mathrm{TiO}_{2}$ enrichment and LC-MS/MS analysis. The procedures for in gel digestion, sample preparation, and data analysis were as previously described [22].

\section{Statistical analysis}

Results from all experiments, unless specified, were analyzed by student's $t$ test for a statistical significant difference.

\section{Authors' Contributions}

X.Q. performed animal studies, colony formation and viability assays, in vitro and in vivo Hsp90 phosphorylation experiments, Hsp90 sequence analysis and generating mutated Hsp90 constructs and cloning them into pcDNA3 vector, conducted K-Ras rescued experiments, stable transfection and infection experiments, established Ras transformed MEF cell lines, and performed transient transfection for protein-protein interaction by IPs/WB, and protein stability experiments. C.X. collected pathological specimens, performed IHC staining and patient survival analysis. S.H., G.C., M. C., and S.P.M. designed and/or performed proteomic analysis, and S.H. also generated Flag-p38 $\gamma$ stably expressed 116 and Hke-3 cell lines and p38 $\gamma$ depleted 116 cells, performed associated IP/WB analysis, and WB in a panel of colon cancer cells. N.Y. participated in colon cancer xenograft studies. G.L. and L.D. formed IHC staining, scoring, and statistical analysis. A.S. contributed to patient survival data analysis and S.T. assisted in manuscript editing, whereas Z.B. participated in pathological analysis and data interpretation. S.W. supervised pathological studies and was responsible for designing clinical experiments and patient survival data analysis. G.C. was responsible for designing all other experiments, performed animal studies, supervised overall projects, provided funding supports, interpreted results, and wrote the manuscript.

\section{ACKNOWLEDGEMENTS}

We would like to thank Drs. Jiahuai Han, Len Neckers, Carol Williams, Ana Cuenda, and Michael Dwindle for providing essential reagents that made this work possible. This study was in part supported by grants from Department of Veterans Affairs (Merit Review), and Cancer Center of Medical College of Wisconsin (G.C.), and an Innovation Research Grant from Biotechnology \& Bioengineering Center of Medical College of Wisconsin (S.H. and G.C.).

\section{REFERENCES}

1. Downward J: Targeting Ras signalling pathways in cancer therapy. Nature Rev Cancer 2003, 3:11-22.

2. Prior IA, Lewis PD, Mattos C: A comprehensive survey of ras mutations in cancer. Cancer Res 2012, 72:2457-2467.

3. Malumbres M, Barbacid M: Ras oncogenes: the first 30 years. Nature Rev Cancer 2003, 3:7-13.

4. Gysin S, Salt M, Young A, McCormick F: Therapeutic strategies for targeting Ras proteins. Genes \& Cancer 2011, 2:359-372. 
5. Tanaka M, Omura K, Watanabe Y, Oda Y, Nakanishi I: Prognostic factors of colorectal cancer: K-ras mutation, overexpression of the p53 protein and cell proliferative activity. J Surg Oncol 1994, 57:57-64.

6. Khambata-Ford S, Garrett CR, Neropol NJ, Basik M, Harbison $\mathrm{CT}$, Wu S, Wong TW, Huang X, Takimoto $\mathrm{CH}$, Godwin $\mathrm{AK}$ et al: Expression of epiregulin and amphiregulin and K-ras mutation status predict disease control in metastatic colorectal cancer patients treated with cetuximab. J Clin Oncol 2007, 25:3230-3237.

7. Karapetis CS, Khambata-Ford S, Jonker DJ, O'Callaghan CJ, Tu D, Tebbutt NC, Simes RJ, Chalchal H, Shapiro JD, Robitaille $\mathrm{S}$ et al: K-ras mutations and benefit from Cetuximab in advanced colorectal cancer. N Engl J Med 2008, 359:1757-1765.

8. Little AS, Balmanno K, Sale MJ, Newman S, Dry JR, Hampson M, Edwards PAW, Smith PD, Cook SJ: Amplification of the driving oncogene, KRAS or BRAF, underpins acquired resistance to MEK1/2 inhibitors in colorectal cancer cells. Sci Signal 2011, 4:ra17.

9. Yoon J, Koo K, Choi K: MEK1/2 inhibitors AS703026 and AZ6244 may be potential therapies for KRAS mutated colorectal cancer that is resistant to EGFR monoclonal antibody therapy. Cancer Res 2011, 71:445-553.

10. Blagosklonny MV: Hsp90-90-associated oncoproteins: multiple targets of geldanamycin and its analogs. Leukemia 2002, 16:455-462.

11. Xu W, Trepel J, Neckers L: Ras, ROS and proteltoxic stress: a delicate balance. Cancer Cell 2011, 20:281-282.

12. Scaltriti M, Dawood S, Cortes J: Molecular pathways: targeting Hsp90-who benefits and who does not. Clin Cancer Res 2012, 18:4508-4513.

13. Karnoub AE, Weinberg RA: Ras oncogenes: split personalities. Nat Rev Mol Cell Biol 2008, 9:517-531.

14. Chen G, Hitomi M, Han J, Stacey DW: The p38 pathway provides negative feedback to Ras proliferative signaling. J Biol Chem 2000, 275:38973-38980.

15. Bulavin DV, Demidov ON, Saito S, Kauraniemi P, Phillips C, Amundson SA, Ambrosino C, Sauter G, Nebreda AR, Anderson CW et al: Amplification of PPM1D in human tumors abrogates p53 tumor-suppressor activity. Nature Genet 2002, 31:210-215.

16. Dolado I, Swat A, Ajenjo N, De Vita G, Cuadrado A, Nebreda AR: p38a MAP kinase as a sensor of reactive oxygen species in tumorigenesis. Cancer Cell 2007, 11:191205.

17. Tang J, Qi X, Mercola D, Han J, Chen G: Essential role of $\mathrm{p} 38 \mathrm{~g}$ in K-Ras transformation independent of phosphorylation. J Biol Chem 2005, 280:23910-23917.

18. Qi X, Tang J, Loesch M, Pohl N, Alkan S, Chen G: p38g MAPK integrates signaling cross-talk between Ras and estrogen receptor to increase breast cancer invasion. Cancer Res 2006, 66:7540-7547.

19. Qi X, Pohl NM, Loesch M, Hou S, Li R, Qin JZ, Cuenda
A, Chen G: p38a antagonizes p38g activity through c-Jundependent ubiquitin-proteasome pathways in regulating Ras transformation and stress response J Biol Chem 2007, 282:31398-31408.

20. Hou SW, Zhi H, Pohl N, Loesch M, Qi X, Li R, Basir Z, Chen G: PTPH1 dephosphorylates and cooperates with p38g MAPK to increases Ras oncogenesis through PDZmediated interaction. Cancer Res 2010, 70:2901-2910.

21. Kyriakis JM, Avruch J: Mammalian MAPK signal transduction pathways activated by stress and inflammation: a 10-year update. Physiol Rev 2012, 92:689-737.

22. Hou S, Padmanaban S, Qi X, Leep A, Mirza S, Chen G: p38g MAPK signals through phosphorylating its phosphatase PTPH1 in regulating Ras oncogenesis and stress response. J Biol Chem 2012, 287:27895-27905.

23. Loesch M, Zhi H, Hou S, Qi X, Li R, Basir Z, Iftner T, Cuenda A, Chen G: p38g MAPK cooperates with c-Jun in trans-activating matrix metalloproteinase 9. J Biol Chem 2010, 285:15149-15158.

24. Qi X, Zhi H, Lepp A, Wang P, Huang J, Basir Z, Chitambar CR, Myers CR, Chen G: p38g mitogen-activated protein kinase (MAPK) confers breast cancer hormone sensitivity by switching estrogen receptor (ER) signaling from classical to nonclassical pathway via stimulating ER phosphorylation and c-Jun transcription. J Biol Chem 2012, 287:1468114691.

25. Rosenthal DT, Lyer H, Escudero S, Bao L, Wu Z, Ventura AC, Kleer CG, Arruda EM, Garikipati K, Merajver SD: p38g promotes breast cancer motility and metastasis through regulation of RhoC GTPase, cytoskeletal architecture, and a novel leading edge behavior. Cancer Res 2011, 71 63386349

26. Meng F, Zhang H, Liu G, Kreike B, Chen W, Sethi S, Miller F, Wu G: p38g mitogen-activated protein kinase contributes to oncogenic properties maintenance and resistance to poly (ADP-ribose)-polymerase-1 inhibition in breast cancer Neoplasia 2011, 13:472-482.

27. Liu L, Liu Z, Jiang H, Zhang W, Qi S, Hu J, Wang X, Wu M: Gene expression profiles of hepatoma cell line HLE. World J Gastroenterol 2003, 9:683-687.

28. Yang K, Liu Y, Liu Z, Liu Z, Liu X, Chen X, Zeng Y: p38g overexpression in gliomas and its role in proliferation and apoptosis. Scientific Rep 2013, 3:1-5 DOI: 10.1038/ srep02089.

29. Qi X, Hou S, Lepp A, Li R, Basir Z, Lou Z, Chen G: Phosphorylation and stabilization of topoisomerase IIa by p38g MAPK sensitize breast cancer cells to its poisons. J Biol Chem 2011, 286:35883-35890.

30. Trepel J, Mollapour M, Giaccone G, Neckers L: Targeting the dynamic HSP90 complex in cancer. Nat Rev Cancer 2010, 10:537-549.

31. Taipale M, Jarosz D, Lindquist S: HSP90 at the hub of protein homeostasis: emerging mechanistic insights. Nat Rev Mol Cell Biol 2010, 11:515-528. 
32. Schulte TW, Blagosklonny MV, Ingui C, Neckers L: Disruption of the Raf-1-Hsp90 molecular complex results in destablization of Raf-1 and loss of Raf-1-Ras association. J Biol Chem 1995, 270:24585-24588.

33. Whitesell L, Mimnaugh EG, Costa B, Myers CE, Neckers L: Inhibition of heat shock protein Hsp90-pp60v-src heteroprotein complex formation by benzoquinone ansamycins: essential role for stress protein in oncogenic transformation. Proc Natl Acad Sci USA 1994, 91:83248328.

34. Dias SDR, Friedlos F, Light Y, Springer C, Workman P, Marais R: Activated B-RAF is an Hsp90 client protein that is targeted by the anticancer drug 17-allylamino-17demethoxygeldanamycin. Cancer Res 2005, 65:1068610691.

35. Grbovic OM, Basso AD, Sawai A, Ye Q, Friedlander P, Solit D, Rosen N: V600E B-Raf requires the Hsp90 chaperone for stability and is degraded in response to Hsp90 inhibitors. Proc Natl Acad Sci USA 2006, 103:57-62.

36. Kamal A, Sensintaffar J, Zhang L, Boehm MF, Fritz LC, Burrows FJ: A high-affinity conformation of Hsp90 confers tumor selectivity on Hsp90 inhibitors. Nature 2003, 425:407-410.

37. Blagosklonny MV: Matching targets for selective cancer therapy. Drug Discov Today 2003, 8:1104-1107.

38. Khalil A, Kabapy NF, Deraz SF, Smith C: Heat shock proteins in oncology: diagnostic biomarkers or therapeutic targets? Biochem Biophy Acta 2011, 1816:89-104.

39. Mollapour M, Tsutsumi S, Donnelly AC, Beebe K, Tokita MJ, Lee M, Lee S, Morra G, Bourboulia D, Scroggins BT et al: Swe1Wee1-dependent tyrosine phosphorylation of Hsp90 regulates distict facets of chaperone function. Mol Cell 2010, 37:333-343.

40. Li Z, Jiang Y, Ulevitch RJ, Han J: The primary structure of p38g: a new member of p38 group of MAP kinases. Biochem Biophys Res Commun 1996, 228:334-340.

41. Mollapour M, Tsutsumi S, Truman AW, Xu W, Vaughan CK, Beebe K, Konstantinova A, Vourganti S, Panaretou B, Piper PW et al: Threonine 22 phosphorylation attenuates hsp90 interaction with cochaperones and affects its chaperone activity. Mol Cell 2011, 41:672-681.

42. Shirasawa S, Furuse M, Yokoyama N, Sasazuki T: Altered growth of human colon cancer cell lines disrupted at activated Ki-ras. Science 1993, 260:85-88.

43. Matallanas D, Romano D, Al-Mulla F, O’Neill E, Al-Ali W, Crespo P, Doyle B, Nixon C, Sansom O, Drosten M et al: Mutant K-Ras activation of the proapoptotic MST2 pathway is antagonized by wild-type K-Ras. Mol Cell 2011, 44:893-906.

44. Qi X, Tang J, Pramanik R, Schultz RM, Shirasawa S, Sasazuki T, Han J, Chen G: p38 MAPK activation selectively induces cell death in K-ras mutated human colon cancer cells through regulation of vitamin D receptor. J Biol Chem 2004, 279:22138-22144.
45. Mollapour M, Neckers L: Post-translational modifications of Hsp90 and their contributions to chaperone regulation. Bioch Biophy Acta 2012, 1823:648-655.

46. Songyang Z, Lu KP, Kwon YT, Tsai L, Filhol O, Cantley LC: A stuctural basis for substrate specificites of protein ser/thr kinases: primary sequence preference of casei kinases 1 and II, NIMA, phosphrylase kinase, calmodulindependent kinase II, CDK5, and Erk1 Mol Cell Biol 1996, 16:6486-6493.

47. Ozes O, Blatt LM, Seiwert SD: Use of pirfenidone in therapeutic regimens. United States Patent-US 7,407,973 B2 2008, Aug. 5th:1-46.

48. Cain WC, Stuart RW, Lefkowitz DL, Starnes JD, Margolin $\mathrm{S}$, Lefkowitz SS: Inhibition of tumor necrosis factor and subsequent endotoxin shock by pirfenidone. Int $\mathrm{J}$ Immunopharmacol 1998, 20:685-695.

49. Richeldi L, Yasothan U, Kirkpatrick DS: Pirfenidone. Nature Reviews/Drug Disconvery 2011, 10:489-490.

50. Noble PW, Albera C, Bradford W, Costabel U, Glassberg MK, Kardatzke D, King TE, Lancaster Jea: Pirfenidone in patients with idiopathic pulmonary fibrosis (CAPACITY): two randomised trials. Lancet 2011, 377:1760-1769.

51. Schaefer CJ, Ruhrmund DW, Pan L, Selwert SD, Kossen K: Antifibrotic activities of pirfenidone in animal models. Eur Respir Rev 2011, 20:85-97.

52. Moran N: p38 kinase inhibitor approved for idiopathic pulmonary fibrosis. Nat Biotechnol 2011, 29:301.

53. Blagosklonny MV: Overcoming limitations of natural anticancer drugs by combining with artificial agents. Trends Pharmacological Sci 2005, 26:77-81.

54. Demidenko ZN, An WG, Lee JT, Romanova LY, McCubrey JA, Blagosklonny MV: Kinase-addiction and biphasic sensitity-resistance of bcr-abl and raf-1-expressing cells to imatinib and geldanamycin Cancer Biol Ther 2005, 4:484-490.

55. Dogan T, Harms GS, Hekman M, Karreman C, Oberoi TK, Alnemri ES, Rapp UR, Rajalingam K: X-linked and cellular IAPs modulate the stability of C-RAF kinase and cell motility. Nat Cell Biol 2008, 10:1447-1455.

56. Chen G, Oh S, Monia BP, Stacey DW: Antisense oligonucleotides demonstrate a dominant role of c-Ki-RAS proteins in regulating the proliferation of diploid human fibroblasts. J Biol Chem 1996, 271:28259-28265.

57. Kopp F, Wagner E, Roidl A: The proto-oncogene KRAS is targeted by mtR-200c. Oncotarget 2014, 5:185-195.

58. Blagosklonny MV, Toretsky J, Bohen S, Neckers L: Mutant conformation of p53 translated in vitro or in vivo requires functional Hsp90. Proc Natl Acad Sci USA 1996, 93:83798363.

59. Demidenko ZN, Vivo C, Halicka HD, Li CJ, Bhalla K, Brounde EU, Blagosklonny MV: Pharmacological induction of Hsp70 protects apoptosis-prone cells from doxorubicin: comparison with caspase-inhibitor- and cyclearrest-mediated cytoprotection. Cell Death Differ 2006, 
13:1434-1441.

60. Azoitei N, Hoffmann CM, Ellegast JM, Ball CR, Obermayer K, GoBele Uea: Targeting of KRAS mutant tumors by HSP90 inhibitors involves degradation of STK33. J Exp Med 2012, 209:697- 